\title{
Improving the case detection of pulmonary tuberculosis by bleach microscopy method in the North West of Nigeria
}

\author{
Ameh James ${ }^{1 \star}$, Shuaibu Uba Abba ${ }^{2}$, Aminu Ibrahim ${ }^{3}$, Henry Mbah $^{4}$, Humphrey Musuluma ${ }^{4}$, \\ Kingsley Ochei ${ }^{4}$, Saffiatou Darboe ${ }^{5}$ and Kwasi Torpey ${ }^{4}$ \\ ${ }^{1}$ Family Health International 360, Plot 1073-A1 Godab Plaza J.S. Tarka Street Area 3, Garki, Abuja, FCT, Nigeria. \\ ${ }^{2}$ Federal Medical Centre, Gusau, Zamfara State, Nigeria. \\ ${ }^{3}$ Sir Yaya Memorial Hospital, Birni-Kebbi, Kebbi State, Nigeria. \\ ${ }^{4}$ Family Health International 360, Plot 1073-A1 Godab Plaza J.S. Tarka Street Area 3, Garki, Abuja, FCT, Nigeria. \\ ${ }^{5}$ Medical Research Council Unit, The Gambia.
}

Accepted 24 July, 2013

\begin{abstract}
Microscopy of direct smears for acid-fast bacilli (AFB) remains the common method for the laboratory diagnosis of pulmonary tuberculosis in most laboratories in our setting. The bleach microscopy method has been introduced and evaluated in different settings as an alternative to direct smear microscopy. However, this approach is yet to be evaluated in a typical government owned/public health laboratory in our setting thus the need for this study. This study was conducted in two Family Health International (FHI) supported health facilities in North West Nigeria. A total of 1075 sputum specimens were collected for the study, comprising of new and follow up cases, regardless of age group and sex. The sputa were examined by two methods, direct smear microscopy and household bleach and centrifugation method. Out of the 1075 sputum specimens, 171 specimens (40.3\%) were positive for AFB by direct microscopy and $253(59.7 \%)$ were positive for AFB by the household bleach method. There was a significant increase in the number of AFB positive specimens by the household bleach method $(p<0.05)$. The bleach method significantly increase tuberculosis (TB) case detection compared to direct smear microscopy. The National TB and Leprosy Control Program could consider supporting the use of this method.
\end{abstract}

Key words: Bleach microscopy, tuberculosis, Nigeria, laboratory.

\section{INTRODUCTION}

Accurate and reliable laboratory diagnosis of Mycobacterium tuberculosis in low resource setting still remains a challenge. This challenge is further aggravated in human immunodeficiency virus (HIV)-associated tuberculosis (Perkins, 2007; Getahun et al., 2007; Havlir et al., 2008; Uys et al., 2007). The common method of diagnosis is direct sputum smear microscopy and this method is widely available in most laboratories in our setting. This method has been shown to have low sensitivity (22 to $78 \%$ ) and high specificity (93.3 to 99.9\%) (Pfaller, 1994). Inspite of the low sensitivity, the method remains the cornerstone of laboratory diagnosis of tuberculosis because it is inexpensive, rapid and highly specific in settings where tuberculosis is endemic (Maryline et al., 
2008; Cattamanchi et al., 2010). Different diagnostic methods have been evaluated in order to improve the detection of tuberculosis in the laboratory, including serologic technique (Walid et al., 2011), nucleic acid amplification (Catharina et al., 2010) and optimizing smear microscopy (Ongkhammy et al., 2009; Maryline et al., 2008; Wah et al., 2001; Farniaet al., 2002; Frimpong et al., 2005; Merid et al., 2009). Among these evaluations, optimizing smear microcopy with the use of bleach or sodium hypochlorite $(\mathrm{NaOCl})$ seems to be the choice in low resource settings with high burden of tuberculosis (TB). The bleach microscopy methods evaluated by different workers employed concentration methods like centrifugation and sedimentation using different bleach concentrations. Overall, the bleach microscopy employs digestion of sputum with bleach, followed by a concentration step, before the smear preparation for Ziehl-Neelsen (ZN) staining (Gebre-Selassie, 2003; Wilkinson et al., 1997; Miorner et al., 1996)

Of the two evaluated bleach methods, those that used locally obtained domestic bleach and sedimentation method at room temperature appear to be inexpensive and suitable for primary and secondary laboratories in low income countries (Maryline et al., 2008). However, due to overnight sedimentation required, we set out to use centrifugation so that, same day result can be achieved. Therefore, we evaluated the use of domestic bleach $(5 \%)$ and centrifugation among patients with suspected tuberculosis in a secondary and tertiary health facility. Our aim primarily was to determine the gain in positivity of microscopic detection in a setting where resources are limited.

Although the bleach microscopy method has been introduced and evaluated in different settings using different bleach preparation and concentration methods, however this approach is yet to be evaluated in a typical government owned/public health laboratory in our setting, thus the need for this study.

\section{METHODOLOGY}

\section{Settings and patients}

Participants were drawn from two urban public health facilities supported by Family Health International; these facilities are regarded by the public as referral centres. One of the centres is a tertiary health institution, Federal Medical Centre located in Gusau, Zamfara State, while the other centre is known as Sir Yaya Memorial Hospital in Birnin Kebbi, Kebbi State. The participants were recruited with their informed consent from the Direct Observation Therapy (DOT) clinics of the hospitals and inclusion criteria included presentation with cough for more than two weeks and aged more than fifteen years. These patients visited these facilities between May and October, 2011 and they included new and treatment cases.

\section{Sample collection}

For new cases, patients submitted three sputum samples over two consecutive days. The first sample was collected in the laboratory at first visit to the clinic while the second was collected at patient's home early in the morning before mouth brushing and the third sample in the laboratory, when the patient brought the morning sample. Generally, instructions were given on how to produce a good quality sputum sample. The two samples collected in the laboratory were produced by patients in an open and free ventilated area. As controls, we did sample 48 patients as new cases from the out-patient department that were not referred to the DOT clinic but not all the patients were able to produce 3 sputum samples like the new cases referred to the DOT clinic.

\section{Direct smear preparation}

Smears were made from the purulent part of the sputum and heat fixed and stained with ZN method (1\% filtered carbol-fuchsin and $0.1 \%$ methylene blue).

\section{Bleach digestion of sputum and concentration by centrifugation}

The remaining sputum ( 1 to $2 \mathrm{ml}$ ) was transferred into a $15 \mathrm{ml}$ screw capped tube with equal volume of undiluted commercial bleach. This mixture was incubated at room temperature (18 to $35^{\circ} \mathrm{C}$ ) undisturbed for $15 \mathrm{~min}$ and centrifuged for $15 \mathrm{~min}$ at 3,000 revolutions per minute. The supernatant was discarded and pellets suspended in few drops of remaining fluids. A drop of the deposit using a disposable dropper was transferred to a slide. A bleach smear of about 1 to $2 \mathrm{~cm}$ was made and allowed to air-dry, heat fixed and stained by the ZN method.

\section{Microscopic examination and interpretation}

The smears (direct and bleach) were read using oil immersion lens of ordinary light microscope by experienced microscopists who were blinded to their results. For both direct and bleach slides, positive and negative smears were defined according to the National Tuberculosis and Leprosy Control Program (NTBLCP) Acid Fast Bacilli (AFB) grading (Table 1).

\section{RESULTS}

1075 sputum samples were examined, 171 (40.3\%) were positive for acid-fast bacilli (AFB) by direct microscopy. After bleach treatment and centrifugation, the number of smear positive samples was increased to 253 (59.7\%). All direct microscopy smears positive for AFB were also positive by bleach concentration method. The increase in numbers of AFB positive samples by bleach method was significant $(p<0.05)$ (Table 2$)$. Increase in the average number of AFB seen per microscope field in the smears prepared after bleach treatment and concentration was observed. $100(58.5 \%)$ smears graded as + by direct microscopy increased to ++ and $71(41.5 \%)$ graded as ++ increased to +++. The clinical management of identified 82 samples by bleach method indicated that 20 new patients were eligible for enrolment into TB treatment, as they were AFB positive on submission of 2 nd or $3 \mathrm{rd}$ sputum samples while 8 patients were known pulmonary tuberculosis in continuation phase of anti-TB drug treatment but was initially recorded as negative by direct 
Table 1. Guide to AFB microscopy interpretation.

\begin{tabular}{lc}
\hline Number of AFB & Recording/reporting \\
\hline No AFB in at least 100 fields & Negative \\
$1-9$ AFB seen in 100 fields & Actual number \\
$1-9$ AFB in 10 fields & + \\
$1-9$ AFB per field & ++ \\
$>10$ AFB per field & +++ \\
\hline
\end{tabular}

Table 2. Comparing direct microscopy and bleach method.

\begin{tabular}{lccc}
\hline Parameter & \multicolumn{3}{c}{ Bleach processed and concentration } \\
\hline Direct microscopy & Positive & Negative & Total \\
\hline Positive & 171 & 0 & $171(40.3 \%)$ \\
Negative & 82 & 822 & 904 \\
Total & $253(59.7 \%)$ & 822 & 1075 \\
\hline
\end{tabular}

microscopy as per the controls, 125 sputum samples collected from the patients not referred to the DOT clinic tested negative by direct microscopy and bleach method.

\section{DISCUSSION}

The bleach method to the best of our knowledge was applied for the first time in our settings for the case detection of pulmonary TB. It was compared to the direct smear microscopy on a large number of sputum samples from two different sites, one secondary and the other was tertiary. These two facilities are regarded as referral centres by the public because cases that require significant expertise attention at a low cost are handled at these centres. Rather than analyzing the results of our findings separately, we did analyze the results as one entity in order to provide adequate information and these two laboratories are Government owned public health facilities with the same resources. Although, bleach is a household commodity, cheap and lowers the risk of laboratory infection, it can easily disintegrate the bacilli if allowed to act for $\geq 60 \mathrm{~min}$ (Yassin et al., 2003; Wah et al., 2001). As a result, bleach method is meant for only microscopy and not sputum intended for culture.

In our study, the bleach smear microscopy increases the effectiveness of TB case finding, detecting 20 new patients who were originally diagnosed as negative by the direct method. This is similar to another study conducted in Laos (Ongkhammy et al., 2009). This detection was observed on submission of 2 nd or 3rd sputum samples. This indicates that three sputum samples are still necessary to confirm the diagnosis of tuberculosis, unlike the current thinking of using one or two sputum samples. World Health Organization (WHO) had initially described that if only one sample is used for the diagnosis of tuberculosis, nearly $20 \%$ of smear positive patients will be missed. The 2 nd sample will identify most of the remaining patients while the 3rd sample which is obtained at the same time as the 2nd sample is submitted helps to confirm the diagnosis. This view by WHO is beginning to change due to the recent introduction of Xpert MTB/RIF (Catharina et al., 2010). This technology is based on nucleic acid amplification and only one sample is required. However, this technology may not yet be relevant in a setting where the basic amenities/infrastructure required in a medical laboratory are still not available and where they are available, they are already dilapidated. So, for now it is important we identify another approach to improve on the diagnosis of pulmonary TB in a low resource setting.

The major advantage of bleach method is the higher density of AFB per field observed after concentration of the sputum and the reduction of debris present in the sample allowing a clear field for bacteria detection (Wah et al., 2001). This advantage facilitates the examination of the stained smears and reduced the time required for microscopy. This assertion was noted in $58.5 \%$ of smears graded by direct method to be + , increased to ++ by bleach method. Likewise, $41.5 \%$ graded as ++ by the first method, and increased to +++ by the second method. This increase indicates two fold increases in AFB concentration with the use of bleach method. Also, this study was able to identify 8 patients known to be positive for pulmonary tuberculosis in continuation phase but graded as negative by direct method. Again, this is suggestive of the ability of the bleach method to concentrate the bacilli.

In our study, we could not evaluate the sensitivity and specificity of the two methods compared because of the absence of mycobacterial culture. However, the increase in the numbers of AFB positive samples by bleach method was significant $(p<0.05)$. This result confirms those published in ten out of thirteen studies without a gold standard (Angeby et al., 2004). We are also aware of the identified limitations that may affect the implementtation of bleach method by different workers in different settings. Among this limitation is lack of standardization and quality assurance (Ongkhammy et al., 2009) but in our setting, quality assurance of sputum microscopy is a mandatory component of the NTBLCP. Hence, implementing bleach method requires only brief training, which will provide an excellent opportunity to strengthen the procedures in all the NTBLP supported laboratories.

This study did not evaluate the HIV status of the recruited patients but the sentinel survey of seroprevalence of HIV in the region per urban setting where this study was conducted is $2.7 \%$ (Federal Ministry of Health, 2010). So, we did not evaluate the impact of bleach microscopy in TB-HIV co-infection. Further study is required to determine the importance of bleach microscopy among TB-HIV co-infection patients. 


\section{Conclusion}

The introduction of bleach smear microscopy as a routine diagnostic test is feasible and could make a substantial impact on the effectiveness of DOT strategy in our setting where culture is not performed routinely.

\section{REFERENCES}

Angeby KAK, Hoffner SE, Diwan VK (2004). Should the 'bleach microscopy method' be recommended for improved case detection of tuberculosis? Literature review and key person analysis. Int. J. Tuberc. Lung Dis. 8(7):806-815.

Cattamanchi A, Davis JL, Pai M, Huang L, Hopewell PC, Steingart KR (2010). Does bleach processing increase the accuracy of sputum smear microscopy for diagnosing pulmonary tuberculosis? J. Clin. Microbiol. 48(7):2433-2439.

Catharina CB, Pamela N, Doris H, Mark PN, Shubhada S, Fiorella K, Jenny A, Rasim T, Robert B, Roxana R, Ana M, Martin J, Sean MO, David HP, Sabine RG, Eduardo G, Camilla R, David A, Perkins MD (2010). Rapid molecular detection of tuberculosis and rifampin resistance. N. Engl. J. Med. 363(11):1005-1015.

Farnia P, Mohammadi F, Zarifi Z, Tabatabee DJ, Ganavi J, Ghazisaeedi K, Farnia PK, Gheydi M, Bahadori M, Masjedi MR, Velayati AA (2002). Improving sensitivity of direct microscopy for detection of acid-fast bacilli in sputum: use of chitin in mucus digestion. J. Clin. Microbiol. 40:508-511.

Federal Ministry of Health (2010). Technical Report on National HIV Sero-prevalence sentinel survey. p. 16.

Frimpong EH, Adukpo R, Owusu-Darko K (2005). Evaluation of two novel Ziehl-Neelsen methods for tuberculosis diagnosis. West Afr. J. Med. 24:316-320.

Gebre-Selassie S (2003). Evaluation of the concentration sputum smear technique for the laboratory diagnosis of pulmonary tuberculosis. Trop. Doct. 33:160-162.

Getahun H, Harrington M, O'Brien R, Nunn P (2007). Diagnosis of smear negative pulmonary tuberculosis in people with HIV infection or AIDS in resource contrained settings: informing urgent policy changes. Lancet 369:2042-2049.

Havlir DV, Getahun H, Sanne I, Nunn P (2008). Opportunities and challenges for HIV care in overlapping HIV and TB epidemics. JAMA. 300:423-430.

Maryline B, Andrew R, Willie G, Laramie G, Francis V, Philippe JG (2008). Bleach sedimentation. An opportunity to optimize smear microscopy for tuberculosis diagnosis in settings of high prevalence of HIV. CID. 46:1710-1716.
Merid Y, Yassin MA, Yamuah L, Kumar R, Engers H, Aseffa A (2009). Validaiton of bleach treated smears for the diagnosis of pulmonary tuberculosis. Int. J. Tuber. Lung Dis. 13:136-141.

Miorner H, Ganlov G, Yohannes Z, Adane Y (1996). Improved sensitivity of direct microscopy for acid-fast bacilli: sedimentation as an alternative to centrifugation for concentration of tubercle bacilli. J. Clin. Microbiol. 34:3206-3207.

Ongkhammy S, Amstutz, Barennes H, Buisson Y (2009). The bleach method improves the detection of pulmonary tuberculosis in Laos. Int. J. Tuberc. Lung. Dis. 13(9):1124-1129.

Perkins MD, Cunningham $J$ (2007). Facing the crisis: improving the diagnosis of tuberculosis in the HIV era. J. Infect. Dis. 196(1):15-27.

Pfaller MA (1994). Application of new technology to the detection, identification and antimicrobial susceptibility testing of Mycobacteria. Am. J. Clin. Pathol. 101:329-336.

Uys PW, Warren RM, van Helden PD (2007). A threshold value for the time delay to TB diagnosis. PLoS One 2(1):757.

Walid BS, Hedi H, Jalel B (2011). Immunochromatographic IgG/IgM test for rapid diagnosis of active tuberculosis. Clin. Vaccine Immunol. 18(12):2090-2094.

Wah WA, Mar MN, Ti T, Win M (2001). Improved method of direct microscopy for detection of acid-fast bacilli in sputum. Southeast Asian J. Trop. Med. Public Health 32(2):390-393.

Wilkinson D, Sturm AW (1997). Diagnosing tuberculosis in a resource poor setting: the value of sputum concentration. Trans. R. Soc. Trop Med. Hyg. 91:420-421.

Yassin MA, Cuevas LE, Gebrexabher H, Squire SB (2003). Efficacy and safety of short term bleach digestion of sputum in case finding for pulmonary tuberculosis in Ethiopia. Int. J. Tuberc. Lung. Dis. 7:678683. 